\title{
The First Report of Differences in Gut Microbiota Composition between Obese and Normal Weight Iranian Subjects
}

\author{
Fateme Ettehad Marvasti ${ }^{1,2}$, Arfa Moshiri ${ }^{3,4}$, Mina Sadat Taghavi ${ }^{2}$, Soheil Riazi ${ }^{2}$, \\ Majid Taati ${ }^{2}$, Seyedeh Fatemeh Sadati ${ }^{2}$, Azadeh Ghaheri ${ }^{5}$, Morteza Masoomi ${ }^{1,6}$, \\ Farzam Vaziri ${ }^{1,6}$, Abolfazl Fateh $^{1,6}$, Pejman Rohani ${ }^{7}$, Samira Tarashi ${ }^{1}$, Andrea \\ Masotti $^{8}$, Sara Ahmadi Badi ${ }^{1^{*}}$ and Seyed Davar Siadat ${ }^{1,6,9^{*}}$ \\ ${ }^{1}$ Microbiology Research Centre, Pasteur Institute of Iran, Tehran, Iran; ${ }^{2}$ The Islamic Azad University, Science and \\ Research Branch, Tehran, Iran; ${ }^{3}$ Gastroenterology and Liver Diseases Research Center, Research Institute for \\ Gastroenterology and Liver Diseases, Shahid Beheshti University of Medical Sciences, Tehran, Iran; ${ }^{4}$ Laboratory of \\ Experimental Therapies in Oncology, IRCCS Istituto Giannina Gaslini, Genoa, Italy; ${ }^{5}$ Departments of Epidemiology and \\ Reproductive Health, Reproductive Epidemiology Research Center, Royan Institute for Reproductive Biomedicine, \\ ACECR, Tehran, Iran; ${ }^{6}$ Mycobacteriology and Pulmonary Research Department, Pasteur Institute of Iran, Tehran, Iran; \\ ${ }^{7}$ Department of Pediatric Gastroentrology and Hepatology, Mofid Children's Hospital, Shahid Beheshti University of \\ Medical Sciences, Tehran, Iran; ${ }^{8}$ Research Laboratories, Bambino Gesù Children's Hospital-IRCCS, Rome, Italy; \\ ${ }^{9}$ Endocrinology and Metabolism Research Center, Endocrinology and Metabolism Clinical Sciences Institute, Tehran \\ University of Medical Sciences, Tehran, Iran
}

Received 4 February 2019; accepted 17 April 2019; published online 14 December 2019

\begin{abstract}
Background: Obesity is a complex disorder influenced by various genetic and environmental factors. It has been shown that gut microbiota, which colonizes gastrointestinal tract, has a substantial role as an environmental factor in the pathophysiology of obesity. Since the composition of gut microbiota alters with regard to different criteria, such as ethnicity, geographical location, diet, lifestyle, age, and gender, we aimed to determine F/B ratio and the abundance of important gut microbiota members, A. muciniphila, F. prausnitzii, Roseburia, Bifidobacterium, and Prevotella in Iranian obese and normal weight individuals, for the first time. Methods: In this study, 50 normal and 50 obese subjects were recruited and classified based on their BMI into normal weight and obese groups. Stool samples were collected. Following DNA extraction from the samples, qPCR was conducted based on 16s rDNA universal primers. Finally, the correlation between the bacterial abundance and obesity was analyzed by statistical analyses. Results: We observed a significant increase of F/B ratio in the obese group, compared to the normal weight group $(p=0.002)$. Although $A$. muciniphila $(p=0.039)$ and Bifidobacterium $(p=$ $0.049)$ abundance significantly decreased, the abundance of $F$. prausnitzii $(p=0.046)$ significantly elevated with BMI increase in the studied groups. Conclusion: Owing to the importance of the gut microbiota composition in obesity development, determination and targeted restoration of gut microbiota pattern could be valuable in the control and treatment of obesity in certain populations. DOI: 10.29252/ibj.24.3.148
\end{abstract}

Keywords: Bacteroidetes, Bifidobacterium, Faecalibacterium prausnitzii, Firmicutes, Microbiota 


\section{INTRODUCTION}

$\mathrm{O}$ besity is a global health problem due to change in people's life style. Various factors, including genetic and environmental factors, are involved in the pathophysiology of obesity ${ }^{[1-3]}$. Gut microbiota has been known as an important environmental factor for inducing and developing obesity. After birth, the gastrointestinal tract is colonized by a complex and dynamic microbial community, which is called gut microbiota. The composition of gut microbiota depends on multiple factors, including genetic background, mode of delivery, nutrition, antibiotic consumption, physical activity, geographical distribution, ethnicity, age, gender, lifestyle, and others ${ }^{[4-6]}$. This microbial community settles down during 2-3 years of life and consists of bacteria, archaea, protozoa, fungi, and viruses. Bacteria are dominant in this microbial population where the Firmicutes and Bacteroidets phyla make up the most frequency of gut microbiota. Also, Actinobacteria, Proteobacteria, and Verrucomicrobia are constituents of gut microbiota with low frequency ${ }^{[7,8]}$. The gut microbiota and its metabolites have determinative role in health and diseases due to the fact that they have significant potential in host, including regulation of inflammatory responses, energy homeostasis, and glucose/lipid metabolism. Therefore, any change in gut microbiota composition, which is termed dysbiosis, can lead to disruption in host functions and development of metabolic disorders, including obesity and type 2 diabetes . In this regard, determination of altered gut microbiota composition is an inevitable part of etiological recognition of obesity ${ }^{[2,9,10]}$.

Obesity is associated with low-grade inflammation, insulin resistance, increased weight gain, and fat deposition $^{[2,11]}$. It has been documented that high-fat diet induces dysbiosis, which favors the increase of energy harvest from diet, deregulation of immune responses, and metabolic pathways ${ }^{[1,2,12]}$. Hence, to achieve healthy state, the delicate arrangement of gut microbiota composition in the gastrointestinal tract is required. In this regard, many studies have shown that $\mathrm{F} / \mathrm{B}$ ratio increases in obese subjects, and changes in F/B ratio have a significant role in calorie intake and have a direct correlation with obesity ${ }^{[13,14]}$.

Currently, numerous investigations have shown that anaerobic intestinal commensal bacteria such as $A$. muciniphila, F. prausnitzii, Roseburia, Bifidobacterium, and Prevotella have significant role in the gut microbiota-host interactions, including influence on host metabolism and immune system through anti-inflammatory properties. Thus, their relative abundance could be a potential health biomarker ${ }^{[15-19]}$.

As mentioned above, dysbiosis is critical starting point in developing obesity and related complications (type 2 diabetes, cardiovascular disease, nonalcoholic fatty liver disease, etc.). Also, F/B ratio, A. muciniphila, F. prausnitzii, Roseburia, Bifidobacterium, and Prevotella influence the pathophysiology of metabolic disorders. As the gut microbiota is under the influence of different factors such as ethnicity, diet, lifestyle, and geographical distribution, we decided to investigate the relative abundance of these bacteria in obese Iranian population, which would be the first report in this context, to the best of our knowledge. For this purpose, fecal samples from Iranian subjects were collected and analyzed using qPCR based on 16s rDNA gene of targeted bacteria. We aimed to determine the correlation between the abundance of the aforementioned bacteria and BMI among our studied population.

\section{MATERIALS AND METHODS}

\section{Study population}

A total of 100 adult Iranian individuals (aged between 20 and 60 years) were selected for this study during October 2016 to December 2017. The subjects were equally grouped into normal weight group with BMI between 18.5 and $24.9 \mathrm{~kg} / \mathrm{m}^{2}$ and obese group with BMI above $25 \mathrm{~kg} / \mathrm{m}^{2}$. Exclusion criteria included the use of corticosteroids, antibiotics, alcohol, smoking, significant infection, and gastrointestinal diseases.

\section{Fecal sampling and DNA extraction}

Subjects were asked to collect their stool samples in a conventional laboratory plastic container dedicated for fecal sampling. The samples were immediately transferred to the laboratory in cold chain storage. These samples were stored at $-80{ }^{\circ} \mathrm{C}$ (fresh frozen) upon arrival until further processing. DNA was extracted from the samples using QIAamp DNA stool mini kit (Qiagen, Hilden, Germany) according to manufacturer's instructions. The quality and quantity of the extracted DNA was analyzed by agarose gel electrophoresis and NanoDrop ND-8000 (Thermo Scientific, USA), respectively ${ }^{[20]}$.

\section{qPCR analyses}

The abundance of bacteria was analyzed using $\mathrm{qPC}$ based on SYBER green method (LightCycler® 96 SW 1.1; Roche, Germany $)^{[13,20,21]}$. Each $20 \mu 1$ of $\mathrm{qPCR}$ reaction was composed of SYBR Premix Ex Taq II 
Table 1. 16S rRNA gene specific primers for the studied bacterial group/species

\begin{tabular}{|c|c|c|c|}
\hline Target organism & Forward $\left(5^{\prime}\right.$ to $\left.3^{\prime}\right)$ & Reverse (5' to $\left.3^{\prime}\right)$ & Ref. \\
\hline Firmicutes & TGAAACTYAAGGAATTGACG & ACCATGCACCTGTC & $\begin{array}{l}{[13]} \\
\end{array}$ \\
\hline Bacteroidetes & AAACTCAAAKGAATTGACGG & GGTAAGGTTCCTCGCGCTAT & {$[13]$} \\
\hline A.muciniphila & CAGCACGTGAAGGTGGGGAC & CCTTGCGGTTGGCTTCAGAT & [20] \\
\hline F. prausnitzii & GGAGGAAGAAGGTCTTCGG & AATTCCGCCTACCTCTGCACT & {$[21]$} \\
\hline Prevotella & CACCAAGGCGACGATCA & GGATAACGCCYGGACCT & {$[47]$} \\
\hline Roseburia & TACTGCATTGGAAACTGTCG & CGGCACCGAAGAGCAAT & {$[47]$} \\
\hline Bifidobacterium & CTCCTGGAAACGGGTGG & GGTGTTCTTCCCGATATCTACA & {$[48]$} \\
\hline Escherichia coli & CATTGACGTTACCCGCAGAAGAAGC & CTCTACGAGACTCAAGCTTGC & [49] \\
\hline
\end{tabular}

(RR820L; Takara, Japan), $0.5 \mu$ of each of the specific 16s rDNA primers (Table 1), and $1 \mu 1$ of the DNA template. Each qPCR reaction was performed in duplicate using LightCycler ${ }^{\circledR}$ 8-Tube Strips (white; Roche). The amplification program was designed according to the appropriate annealing temperature: 1 cycle of $95{ }^{\circ} \mathrm{C}$ for $60 \mathrm{~s}$, followed by 40 cycles of denaturation at $95{ }^{\circ} \mathrm{C}$ for $5 \mathrm{~s}$, annealing at $55^{\circ} \mathrm{C}$ for 30 $\mathrm{s}$, and extension at $72{ }^{\circ} \mathrm{C}$ for $30 \mathrm{~s}$. Melting curve analysis was carried out after amplification to control the specificity of PCR reaction, followed by 1 cycle at $95^{\circ} \mathrm{C}$ for $5 \mathrm{~s}, 60{ }^{\circ} \mathrm{C}$ for $60 \mathrm{~s}$, and $95^{\circ} \mathrm{C}$ for $1 \mathrm{~s}$.

\section{Standard curve}

The abundance of bacteria was calculated as previously described ${ }^{[22]}$. Briefly, the standard curve was prepared using serial dilutions of DNA from standard strain Escherichia coli. This curve allows us to calculate DNA concentration of each bacterium from fecal samples. The standard curve is graphically represented as a semi-log regression line plot of CT value vs. $\log$ of DNA concentration.

\section{Statistical analyses}

In this study, categorical variables are presented as numbers (percent) and continuous variables as mean \pm SD. Independent $t$-test was employed to assess mean differences between the normal and obese groups. Chisquare analysis was used for qualitative data, and K-S analysis was applied to control the normal distribution of the data. Linear regression model and Pearson's correlation coefficient were performed to determine correlation between BMI and the abundance of two bacteria phyla, Bacteroidetes and Firmicutes. Statistical analyses were conducted using SPSS version 24.0 (SPSS Inc., Chicago, IL, USA). All statistical tests were 2-tailed, and a $p<0.05$ was considered statistically significant.

\section{Ethical statement}

The above-mentioned sampling protocols were approved by the National Institute for Medical Research Development (NIMAD, Tehran, Iran; ethical code: IR.NIMAD.REC.1395.043). Written informed consents were provided by all the patients.

\section{RESULTS}

\section{Demographic characteristics of the study population}

Adult subjects were divided, based on BMI, into two groups: normal weight $(50 \%)$ and obese (50\%). Characteristics of the subjects are shown in Table 2. Obese group consisted of 32 overweight subjects with BMI between 25 and $29.9 \mathrm{~kg} / \mathrm{m}^{2}$ and 18 subjects with BMI above $30 \mathrm{~kg} / \mathrm{m}^{2}$. There were not significant differences in age, gender, and height between the two studied groups.

Table 2. Characteristics of obese and normal weight adults under study

\begin{tabular}{lcc}
\hline Characteristics & Obese & Normal weight \\
\hline Subjects & 50 & 50 \\
Gender (male/female) & $25 / 25$ & $25 / 25$ \\
Age $(\mathrm{y})$ & $38.76 \pm 1.76$ & $38.74 \pm 1.41$ \\
Weight $(\mathrm{kg})$ & $83.92 \pm 1.89$ & $64.8 \pm 1.24$ \\
Height $(\mathrm{m})$ & $1.69 \pm 0.015$ & $1.69 \pm 0.012$ \\
BMI $(\mathrm{kg} / \mathrm{m} 2)$ & $29.36 \pm 0.50$ & $22.4 \pm 0.26$ \\
BMI s.d. score & 3.55 & 1.86 \\
\hline
\end{tabular}


Table 3. Mean abundance of each phylum across each of the BMI categories

\begin{tabular}{lccc}
\hline \multirow{2}{*}{ Phylum } & \multicolumn{2}{c}{ BMI index } & \multirow{2}{*}{ p value } \\
\cline { 2 - 3 } & $\mathbf{1 8 . 5}-\mathbf{2 4 . 9}$ & $\geq \mathbf{2 5}$ & \\
\hline Firmicutes & $6.59 \pm 0.19$ & $7.15 \pm 0.18$ & 0.045 \\
Bacteroidetes & $4.92 \pm 08.0$ & $4.64 \pm 10.0$ & 0.040 \\
F/B & $1.66 \pm 0.18$ & $2.5 \pm 0.19$ & 0.002 \\
\hline
\end{tabular}

\section{F/B ratio}

In Table 3, the mean abundance of Firmicutes, Bacteroidetes, and F/B are presented. The results demonstrated that Firmicutes and Bacteroidetes abundance significantly increased and decreased in the obese group and the control, respectively (Fig. 1). Besides, the $\mathrm{F} / \mathrm{B}$ ratio was significantly higher in the obese $(p=0.002)$ than control group.

\section{Quantification and comparison of important gut microbiota memebers}

The concentrations of A. muciniphila, $F$. prausnitzii, Roseburia, Prevotella, and Bifidobacterium were quantified in fecal samples of the studied groups. The highest and lowest concentrations of targeted bacteria were $8.69 \times 10^{13}$ and $1.88 \times 10^{5} \mathrm{CFU} / \mathrm{g}$ for $A$. muciniphila, $9.71 \times 10^{13}$ and $1.83 \times 10^{7} \mathrm{CFU} / \mathrm{g}$ for $F$. prausnitzii, $2.06 \times 10^{13}$ and $4.95 \times 10^{1} \mathrm{CFU} / \mathrm{g}$ for Roseburia, $7.84 \times 10^{14}$ and $8.69 \times 10^{2} \mathrm{CFU} / \mathrm{g}$ for Prevotella, and $4.52 \times 10^{15}$ and $1.19 \times 10^{1} \mathrm{CFU} / \mathrm{g}$ for Bifidobacterium in normal weight and obese groups, respectively (Table 4). In order to find a correlation between the bacterial abundance and BMI, differences in gut microbiota composition between the two groups were analyzed. Our results demonstrated that $A$. muciniphila relative abundance significantly decreased in parallel with BMI increase in obese vs. normal weight ( $p=0.039$ ) groups. Also, a significant reduction of Bifidobacterium relative abundance $(p=0.049)$ was observed in the obese group. In contrast, there was a significant increase of $F$. prausnitzii relative abundance in the obese compared to the normal weight $(p=0.460)$ subjects. Although the relative abundance of Roseburai ( $p=0.170)$ and Prevotell $(p=0.756)$ increased and decreased with BMI increase, respectively, no significant correlation was found between their frequency and the studied groups (Fig. 2).

\section{DISCUSSION}

The dominant roles of gut microbiota in pathophysiology of obesity has been currently evidenced $^{[23]}$. Several studies have shown that the imbalance of energy homeostasis, low-grade inflammation, and insulin resistance are important determinants, which result from dysbiosis and lead to the negative regulation of host metabolism ${ }^{[24]}$. Due to the fact that gut microbiome exerts crucial functions such as the influence on energy harvest from diet, and anti-inflammatory and metabolism regulation, any change in gut microbiota composition can induce and develop obesity ${ }^{[24,25]}$. According to the importance of gut microbiota composition in obesity and its uniqueness in each population, we studied, for the first time, the differences of $\mathrm{F} / \mathrm{B}$ ratio and the relative abundnce of $A$. muciniphila, F . prausnitzii, Roseburia, Bifidobacterium, and Prevotella in normal weight and obese Iranian subjects.

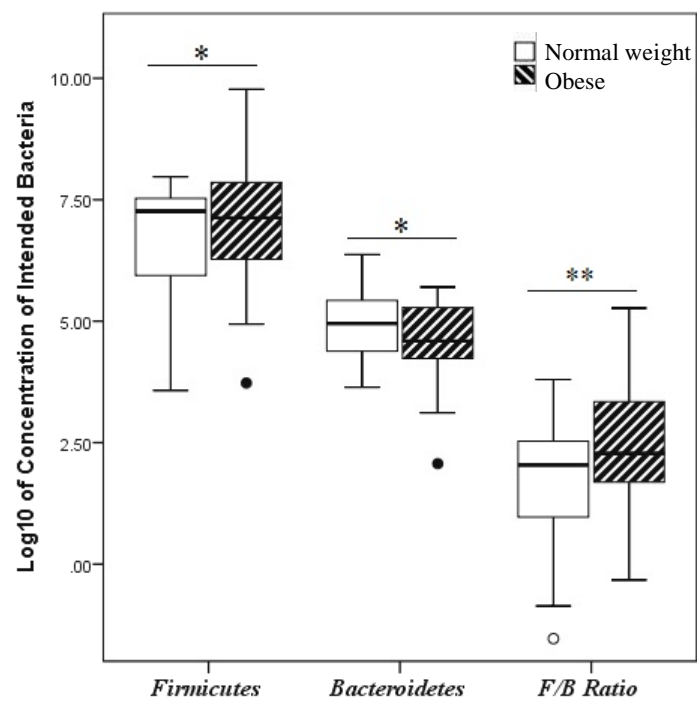

Fig. 1. F/B ratio of obese and normal weight Iranian subjects detected by qPCR. ${ }^{*} p<0.05 ;{ }^{* *} p<0.01$.

Table 4. Relative abundance of A. muciniphila, F. prausnitzii, Roseburia, Prevotella, and Bifidobacterium

\begin{tabular}{lccccc}
\hline & $\begin{array}{c}\text { A. muciniphila } \\
\text { (CFU/g) }\end{array}$ & $\begin{array}{c}\text { F. prausnitzii } \\
\text { (CFU/g) }\end{array}$ & $\begin{array}{c}\text { Roseboria } \\
\text { (CFU/g) }\end{array}$ & $\begin{array}{c}\text { Prevotella } \\
\text { (CFU/g) }\end{array}$ & $\begin{array}{c}\text { Bifidobacterium } \\
\text { (CFU/g) }\end{array}$ \\
\hline Mean & $1.93 \times 10^{12}$ & $4.90 \times 10^{12}$ & $1.05 \times 10^{12}$ & $4.07 \times 10^{13}$ & $6.69 \times 10^{13}$ \\
Std. Error of Mean & $1.02 \times 10^{12}$ & $1.13 \times 10^{12}$ & $2.44 \times 10^{11}$ & $1.43 \times 10^{13}$ & $4.65 \times 10^{13}$ \\
Std. Deviation & $1.02 \times 10^{13}$ & $1.13 \times 10^{13}$ & $2.44 \times 10^{12}$ & $1.43 \times 10^{14}$ & $4.65 \times 10^{14}$ \\
Minimum & $1.88 \times 10^{5}$ & $1.83 \times 10^{7}$ & $4.95 \times 10^{1}$ & $8.69 \times 10^{2}$ & $1.19 \times 10^{1}$ \\
Maximum & $8.69 \times 10^{13}$ & $9.71 \times 10^{13}$ & $2.06 \times 10^{13}$ & $7.84 \times 10^{14}$ & $4.52 \times 10^{15}$ \\
\hline
\end{tabular}




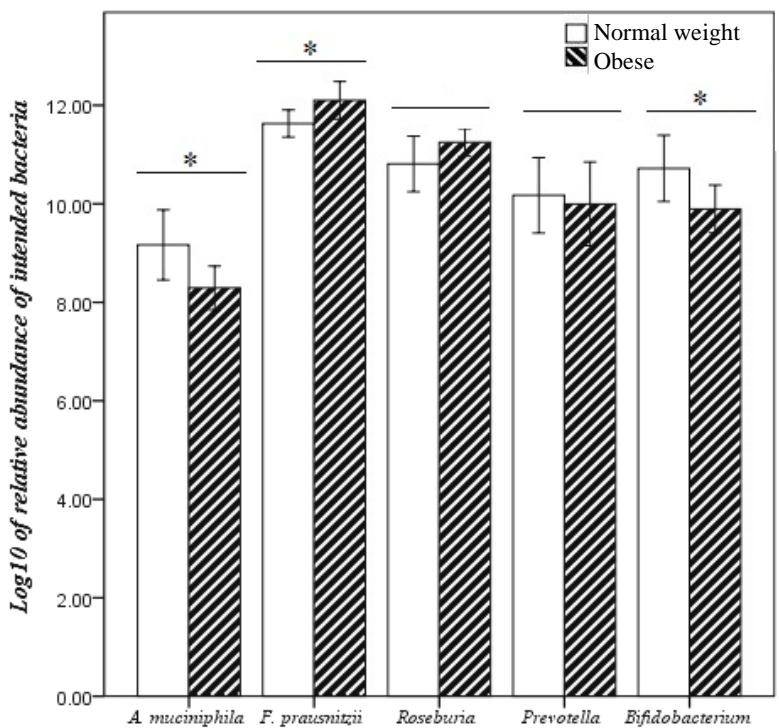

Fig. 2. Comparison of the abundance of important gut microbiota members in normal weight and obese Iranian subjects detected by qPCR. Data of qPCR are expressed as mean $\log _{10} \mathrm{CFU} / \mathrm{g}$. Error bars $95 \%$ CI. ${ }^{*} p<0.05$

Various animal and human studies have revealed the increase of Frimicutes and decrease of Bacteroidets concentrations (increase of F/B ratio) in obese vs. normal subjects ${ }^{[26-29]}$, which is in line with our study. Dominancy of Firmicutes, which is enriched by bacterial genes related to nutrient transporters and primary fermentation enzymes, could be explained by elevated calories absorption and weight gain during obesity ${ }^{[30,-32]}$. Higher frequency of bacterial genes responsible for carbohydrate metabolism, which belongs to Bacteroidetes, has been reported in Turnbaugh et al.'s research of lean and obese-twin gut microbiome ${ }^{[30]}$. Nevertheless, there are inconsistent results of $\mathrm{F} / \mathrm{B}$ ratio. In this regard, Andoh et al.$^{[33]}$ did not observed any difference in the $\mathrm{F} / \mathrm{B}$ ratio between the obese and non-obese Japanese groups.

Recently, the significant roles of some intestinal anaerobic commensal bacteria, such as A. muciniphila, $F$. prausnitzii, Bifidobacterium, Roseburia, and Prevotella, have been illustrated in gut microbiota-host interaction $^{[24,25]}$. One important feature of these bacteria is the production of short-chain fatty acids, which have various functions, including the regulation of gut barrier integrity, regulation of metabolism, and inflammation. Thus, there is a correlation between the abundance of mentioned bacteria and obesity $^{[24,25,34-36]}$.

Increasing body of evidence in animal and human studies has demonstrated an inverse correlation of A. muciniphila abundance with obesity ${ }^{[36,37]}$. In agreement with other studies, A. muciniphila abundance significantly reduced in Iranian obese subjects in comparison with normal weight subjects. This reduction of $A$. muciniphila abundunce is associated with impaired metabolic status during obesity, since it has many health promoting potentials, including regulation of glucose metabolism, blood lipid concentration, and fat distribution ${ }^{[15]}$.

The genus Bifidobacterium has been shown to have beneficial health effects due to the effect on the gut barrier and immune system ${ }^{[38]}$. The association of Bifidobacterium abundance and obesity has been studied in several studies ${ }^{[39-42]}$. Ignacio et al. ${ }^{[43]}$ have reported the negative correlation between the abundance of Bifidobacterium and BMI and showed increased abundance of Bifidobacterium spp. in the lean group. Similarly, we identified significant negative correlation of Bifidobacterium abundance with BMI increase.

There are various studies that inconsistently reported that the $F$. prausnitzii abundance is associated with obesity ${ }^{[44-46]}$. In accordance with the report of gut microbiota composition in obese Indian children ${ }^{[46]}$, the abundance of $F$. prausnitzii significantly increased along with BMI increase in Iranian subjects. However, Feng et al. ${ }^{[45]}$ did not observe any significant difference of $F$. prausnitzii levels between obese and normal Chinese subjects.

Taken together, our results demonstrated a significant increase of $\mathrm{F} / \mathrm{B}$ ratio and reduction of $A$. muciniphila and Bifidobacterium in obese Iranian subjects vs. normal weight individuals. additionally, we observed higher $F$. prausnitzii abundance in obese subjects. Since gut microbiota composition is established based on various factors, including ethnicity, diet, life style, and geographical location, which induce differences between various populations, it is necessary to determine this composition to design proper strategies for obesity treatment in each targeted population.

\section{ACKNOWLEDGEMENTS}

This work was supported by the National Institute for Medical Research Development (NIMAD), Tehran, Iran (IR.NIMAD.REC.1395.043) and Iran Biotech Fund (grant no. 94/10243). The authors would like to thank the colleagues at Mycobacteriology and Pulmonary Research Department and Microbiology Research Center at Pasteur Institute of Iran, Tehran, Iran.

CONFLICT OF INTEREST. None declared. 


\section{REFERENCES}

1. Rampelli S, Candela M, Turroni S, Biagi E, Pflueger M, Wolters M, Ahrens W, Brigidi P. Microbiota and lifestyle interactions through the lifespan. Trends in food science and technology 2016; 57(Part B): 265-272.

2. Cani PD, Delzenne NM. Gut Microbiota, Obesity and Associated Metabolic Disorders. reterieved from: https://www.worldgastroenterology.org/UserFiles/file/ WDHD-2014-handbook-FINAL.pdf

3. Moreno-Indias I, Cardona F, Tinahones FJ, QueipoOrtuño MI. Impact of the gut microbiota on the development of obesity and type 2 diabetes mellitus. Frontiers in microbiology 2014; 5: 190.

4. D'Argenio V, Salvatore F. The role of the gut microbiome in the healthy adult status. Clinica chimica acta 2015; 451(Pt A): 97-102.

5. Rodríguez JM, Murphy K, Stanton C, Ross RP, Kober OI, Juge N, Avershina E, Rudi K, Narbad A, Jenmalm MC, Marchesi JR, Collado MC. The composition of the gut microbiota throughout life, with an emphasis on early life. Microbial ecology in health and disease 2015; 26: 26050 .

6. Sommer F, Bäckhed F. The gut microbiota-masters of host development and physiology. Nature reviews microbiology 2013; 11(4): 227-238.

7. Houghteling PD, Walker WA. Why is initial bacterial colonization of the intestine important to the infant's and child's health? Journal of pediatric gastroenterology and nutrition 2015; 60(3): 294-307.

8. Matamoros S, Gras-Leguen C, Le Vacon F, Potel G, de La Cochetiere MF. Development of intestinal microbiota in infants and its impact on health. Trends in microbiology 2013; 21(4): 167-173.

9. Kasubuchi M, Hasegawa S, Hiramatsu T, Ichimura A, Kimura I. Dietary gut microbial metabolites, short-chain fatty acids, and host metabolic regulation. Nutrients 2015; 7(4): 2839-2849.

10. Lin L, Zhang J. Role of intestinal microbiota and metabolites on gut homeostasis and human diseases. BMC immunology 2017; 18(1): 2.

11. Cox AJ, West NP, Cripps AW. Obesity, inflammation, and the gut microbiota. The lancet diabetes and endocrinology 2015; 3(3): 207-215.

12. Nehra V, Allen JM, Mailing LJ, Kashyap PC, Woods JA. Gut microbiota: modulation of host physiology in obesity. Physiology (Bethesda) 2016; 31(5): 327-335.

13. Koliada A, Syzenko G, Moseiko V, Budovska L, Puchkov K, Perederiy V, Gavalko Y, Dorofeyev A, Romanenko M, Tkach S, Sineok L, Lushchak O, Vaiserman A. Association between body mass index and Firmicutes/Bacteroidetes ratio in an adult Ukrainian population. BMC microbiology 2017; 17(1): 120.

14. Riva A, Borgo F, Lassandro C, Verduci E, Morace G, Borghi E, Berry D. Pediatric obesity is associated with an altered gut microbiota and discordant shifts in $\mathrm{F}$ irmicutes populations. Environmental microbiology 2017; 19(1): 95-105.

15. Dao MC, Everard A, Aron-Wisnewsky J, Sokolovska N, Prifti E, Verger EO, Kayser BD, Levenez F, Chilloux J,
Hoyles L; MICRO-Obes Consortium, Dumas ME, Rizkalla SW, Doré J, Cani PD, Clément K. Akkermansia muciniphila and improved metabolic health during a dietary intervention in obesity: relationship with gut microbiome richness and ecology. Gut 2016; 65(3): 426-436.

16. Hand TW, Vujkovic-Cvijin I, Ridaura VK, Belkaid Y. Linking the microbiota, chronic disease, and the immune system. Trends in Endocrinology and metabolism 2016; 27(12): 831-843.

17. Hippe B, Remely M, Aumueller E, Pointner A, Magnet U, Haslberger AG. Faecalibacterium prausnitzii phylotypes in type two diabetic, obese, and lean control subjects. Beneficial microbes 2016; 7(4): 511-517.

18. Remely M, Tesar I, Hippe B, Gnauer S, Rust P, Haslberger AG. Gut microbiota composition correlates with changes in body fat content due to weight loss. Beneficial microbes 2015; 6(4): 431-439.

19. Schneeberger M, Everard A, Gómez-Valadés AG, Matamoros S, Ramírez S, Delzenne NM, Gomis R, Claret M, Cani PD. Akkermansia muciniphila inversely correlates with the onset of inflammation, altered adipose tissue metabolism and metabolic disorders during obesity in mice. Scientific reports 2015; 5: Article no. 16643.

20. Collado MC, Derrien M, Isolauri E, de Vos WM, Salminen S. Intestinal integrity and Akkermansia muciniphila, a mucin-degrading member of the intestinal microbiota present in infants, adults, and the elderly. Applied and environmental microbiology 2007; 73(23): 7767-7770.

21. Benus RF, van der Werf TS, Welling GW, Judd PA, Taylor MA, Harmsen HJ, Whelan K. Association between Faecalibacterium prausnitzii and dietary fibre in colonic fermentation in healthy human subjects. British journal of nutrition 2010; 104(5): 693-700.

22. Nadkarni MA, Martin FE, Jacques NA, Hunter N. Determination of bacterial load by real-time PCR using a broad-range (universal) probe and primers set. Microbiology 2002; 148(1): 257-266.

23. Tilg H, Moschen AR. Gut Microbiome, Obesity, and Metabolic Syndrome. In: Ahima RS eds. Metabolic Syndrome: A Comprehensive Textbook; 2016. p. 447459.

24. Newsholme P, Homem de Bittencourt PI Jr. Gut associated bacteria are critical to metabolism, inflammation and health. Current opinion in clinical nutrition and metabolic care 2016; 19(4): 245-249.

25. de Clercq NC, Groen AK, Romijn JA, Nieuwdorp M. Gut microbiota in obesity and undernutrition. Advances in nutrition 2016; 7(6): 1080-1089.

26. Barlow GM, Yu A, Mathur R. Role of the gut microbiome in obesity and diabetes mellitus. Nutrition in clinical practice 2015; 30(6): 787-797.

27. Ley RE, Bäckhed F, Turnbaugh P, Lozupone CA, Knight RD, Gordon JI. Obesity alters gut microbial ecology. Proceedings of the national academy of sciences 2005; 102(31): 11070-11075.

28. Mathur R, Barlow GM. Obesity and the microbiome. Expert review of gastroenterology and hepatology 2015; 
9(8): 1087-1099.

29. Sweeney TE, Morton JM. The human gut microbiome: a review of the effect of obesity and surgically induced weight loss. JAMA surgery 2013; 148(6): 563-569.

30. Turnbaugh PJ, Ley RE, Mahowald MA, Magrini V, Mardis ER, Gordon JI. An obesity-associated gut microbiome with increased capacity for energy harvest. Nature 2006; 444(7122): 1027-1031.

31. Krajmalnik- Brown R, Ilhan ZE, Kang DW, DiBaise JK. Effects of gut microbes on nutrient absorption and energy regulation. Nutrition in clinical practice 2012; 27(2): 201-214.

32. Dugas LR, Fuller M, Gilbert J, Layden BT. The obese gut microbiome across the epidemiologic transition. Emerging themes in epidemiology 2016; 13: 2.

33. Andoh A, Nishida A, Takahashi K, Inatomi O, Imaeda H, Bamba S, Kito K, Sugimoto M, Kobayashi T. Comparison of the gut microbial community between obese and lean peoples using $16 \mathrm{~S}$ gene sequencing in a Japanese population. Journal of clinical biochemistry and nutrition 2016; 59(1): 65-70.

34. Cani PD, Everard A, Duparc T. Gut microbiota, enteroendocrine functions and metabolism. Current opinion in pharmacology 2013; 13(6): 935-940.

35. Cani PD, Neyrinck AM, Fava F, Knauf C, Burcelin RG, Tuohy KM, Gibson GR, Delzenne NM. Selective increases of bifidobacteria in gut microflora improve high-fat-diet-induced diabetes in mice through a mechanism associated with endotoxaemia. Diabetologia 2007; 50(11): 2374-2383.

36. Derrien M, Belzer C, de Vos WM. Akkermansia muciniphila and its role in regulating host functions. Microbial pathogenesis 2017; 106: 171-181.

37. Everard A, Belzer C, Geurts L, Ouwerkerk JP, Druart C, Bindels LB, Guiot Y, Derrien M, Muccioli GG, Delzenne NM, de Vos WM, Cani PD. Cross-talk between Akkermansia muciniphila and intestinal epithelium controls diet-induced obesity. Proceedings of the national academy of sciences 2013; 110(22): 90669071.

38. AArboleya S, Watkins C, Stanton C, Ross RP. Gut bifidobacteria populations in human health and aging. Frontiers in microbiology 2016; 7: 1204.

39. Million M, Maraninchi M, Henry M, Armougom F, Richet H, Carrieri P, Valero R, Raccah D, Vialettes B, Raoult D. Obesity-associated gut microbiota is enriched in Lactobacillus reuteri and depleted in Bifidobacterium animalis and Methanobrevibacter smithii. International journal of obesity (London) 2012; 36(6): 817-825.

40. Salazar N, Dewulf EM, Neyrinck AM, Bindels LB, Cani PD, Mahillon J, de Vos WM, Thissen JP, Gueimonde
M, de Los Reyes-Gavilán CG, Delzenne NM. Inulintype fructans modulate intestinal Bifidobacterium species populations and decrease fecal short-chain fatty acids in obese women. Clinical nutrition 2015; 34(3): 501-507.

41. Kondo S, Xiao JZ, Satoh T, Odamaki T, Takahashi S, Sugahara H, Yaeshima T, Iwatsuki K, Kamei A, Abe K. Antiobesity effects of Bifidobacterium breve strain B-3 supplementation in a mouse model with high-fat dietinduced obesity. Bioscience, biotechnology, and biochemistry 2010; 74(8): 1656-1661.

42. QQueipo-Ortuño MI, Seoane LM, Murri M, Pardo M,Gomez-Zumaquero JM, Cardona F, Casanueva F, Tinahones FJ. Gut microbiota composition in male rat models under different nutritional status and physical activity and its association with serum leptin and ghrelin levels. PLoS one 2013; 8(5): e65465.

43. Ignacio A, Fernandes MR, Rodrigues VA, Groppo FC, Cardoso AL, Avila-Campos MJ, Nakano V. Correlation between body mass index and faecal microbiota from children. Clinical microbiology and infection 2016. 22(3): 258.e1-e8.

44. Ryan PM, Delzenne NM. Gut microbiota and metabolism; The gut-brain axis 2016; 2016: 391-401.

45. Feng J, Tang H, Li M, Pang X, Wang L, Zhang M, Zhao Y, Zhang X, Shen J. The abundance of fecal Faecalibacterium prausnitzii in relation to obesity and gender in Chinese adults. Archives of microbiology 2014; 196(1): 73-77.

46. Balamurugan R, Chittaranjan SP, Chandragunasekaran AM, Ramakrishna BS. Molecular detection of the ruminal bacterium, Butyrivibrio fibrisolvens, in feces from rural residents of southern India. Microbial ecology in health and disease 2009; 21(1): 38-43.

47. Larsen N, Vogensen FK, van den Berg FW, Nielsen DS, Andreasen AS, Pedersen BK, Al-Soud WA, Sørensen SJ, Hansen LH, Jakobsen M. Gut microbiota in human adults with type 2 diabetes differs from non-diabetic adults. PLoS one 2010; 5(2): e9085.

48. Matsuki T, Watanabe K, Fujimoto J, Kado Y, Takada T, Matsumoto K, Tanaka R. Quantitative PCR with 16S rRNA-gene-targeted species-specific primers for analysis of human intestinal bifidobacteria. Applied and environmental microbiology 2004; 70(1): 167-173.

49. Bartosch S, Fite A, Macfarlane GT, McMurdo MET. Characterization of bacterial communities in feces from healthy elderly volunteers and hospitalized elderly patients by using real-time PCR and effects of antibiotic treatment on the fecal microbiota. Applied and environmental microbiology 2004; 70(6): 3575-3581. 